\title{
Empirical Modeling of Steering System for Autonomous Vehicles
}

\author{
Ju-Young Kim*, Kyungdeuk Min** and Young Chol Kim ${ }^{\dagger}$
}

\begin{abstract}
To design an automatic steering controller with high performance for autonomous vehicle, it is necessary to have a precise model of the lateral dynamics with respect to the steering command input. This paper presents an empirical modeling of the steering system for an autonomous vehicle. The steering system here is represented by three individual transfer function models: a steering wheel actuator model from the steering command input to the steering angle of the shaft, a dynamic model between the steering angle and the yaw rate of the vehicle, and a dynamic model between the steering command and the lateral deviation of vehicle. These models are identified using frequency response data. Experiments were performed using a real vehicle. It is shown that the resulting identified models have been well fitted to the experimental data.
\end{abstract}

Keywords: Autonomous vehicle, Steering system, Automatic steering Control, Identification, Frequency response based modeling

\section{Introduction}

Automatic steering control of vehicles has been investigated for both autonomous vehicles (AV) [1-3] and driver steering assistance $[4,5]$. Driver steering assistance systems under development include collision avoidance systems, adaptive cruise control, and lane departure avoidance systems. The design of an automatic steering system requires a mathematical model that describes the lateral dynamic motion of the vehicle relative to the steering angle input. A variety of lateral dynamic models have been presented $[1,6-8]$. These models are theoretically derived and are described in the form of nonlinear state equations that include a number of physical parameters. Additionally, linear approximate models are often used for the design of steering controllers. These models generally establish inputs as the steering angle of the wheels and the road curvature and output as the lateral deviation to the lane centerline at a look-ahead distance.

The main difficulty with this approach is the precise identification of some of the physical parameters, such as, the location of the center of gravity, cornering stiffness, and moment of inertia. Moreover, these values are dependent upon tire model and susceptible to changes in payload.

In this paper, we deal with empirical modeling of the steering system for an $\mathrm{AV}$. We consider that the steering system of an AV consists of a steering handle actuator driven by a DC servo-motor, electric power assisted steering (EPAS), a column, gear, wheels, etc. We suppose

$\dagger$ Corresponding Author: School of Electronic Eng., Chungbuk National University, Korea. (yckim @cbu.ac.kr)

* Chungbuk Engineering Team, LG Uplus Corp., Korea. (procit@lguplus.co.kr)

** ADAS Engineering Design Team, Research \& Development Division, Hyundai Motor Group, Korea. (kdmin@hyundai.com)

Received: February 2, 2016; Accepted: December 26, 2016 that the dynamics of the steering system is linear at a fixed operating condition, which is the vehicle speed.

This steering system is represented by three transfer function models; a steering actuator model $\left(G_{a}\right)$ from the steering command input to the steering angle of the steering shaft, a dynamic model $\left(G_{y}\right)$ between the steering angle and the yaw rate of the vehicle body, and an overall steering model $(G)$ from the steering command input to the lateral deviation of vehicle. These models are identified using frequency response data. Frequency responses were obtained through experimental tests on a real vehicle, which is an SUV adapted as an AV [7]. To obtain the pertinent response data, we first present a simple scheme that estimates the location and orientation of the vehicle individually from the measurement data using GPS, a gyroscope, and acceleration sensors.

It is shown that the resulting identified models fits the measured data well.

\section{Experimental Set-Up for Steering System Modeling}

The test vehicle is an SUV (Tucson ix, 2010, Hyundai Motor Co.) as shown in Fig. 1. It has been adapted as an autonomous vehicle by using various actuators and sensors including GPS, LIDAR, cameras, laptop computers etc. [7]. It is possible for us to examine this vehicle under the following operating conditions: (i) the vehicle can keep a predetermined constant speed; and (ii) steering commands of sinusoidal form with various frequencies can be applied to the steering wheel. The experimental set-up for the frequency response test is shown in Fig. 2.

A software tool with LabVIEW (National Instruments Corp.) was developed so that one can set sinusoidal inputs 


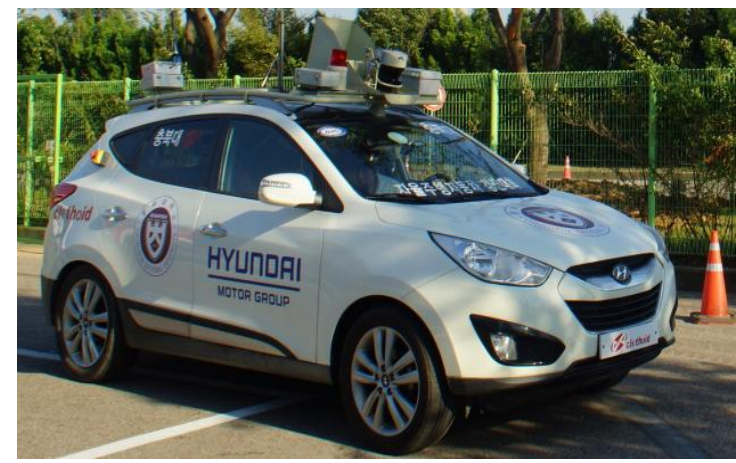

Fig. 1. Test vehicle

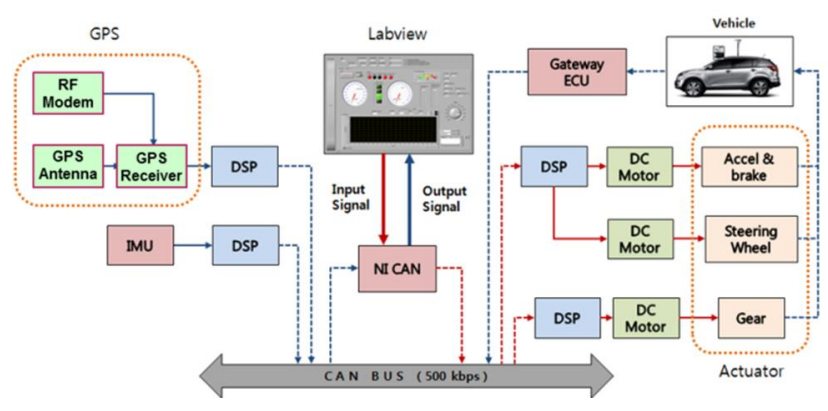

Fig. 2. Experimental set-up for steering system test

for reference steering angle and so that various data transmitted from the vehicle via CAN bus can be collected in real time. Vehicle speed, the yaw rate of the vehicle body, and the steering angle at the column are received from the vehicle itself through the gateway ECU. The location of the vehicle while it moves is measured using a differential global positioning system (DGPS) and an inertial measurement unit (IMU). The horizontal position error of DGPS with RTK mode used here is less than $1 \mathrm{~cm}$.

Experimental tests for the steering system modeling were carried out on the test track of the Korea Automotive Technology Institute (KATECH) in South Korea.

\section{Steering System Modeling}

\subsection{Linear model structures of steering system}

The steering system involves mechanical and electrical components as shown in Fig. 3. The steering wheel has been adapted to be controlled automatically by a DC motor drive. This steering actuator has a feedback controller that makes the steering wheel angle track the reference steering angle. This subsystem is modeled by a transfer function, $G_{a}(\mathrm{~s})$.

In [5], assuming a constant longitudinal velocity, the linearized dynamic bicycle model from the steering input to vehicle lateral motion was represented by a second-order state equation. We describe this linear model as black-box type input-output models as shown in Fig. 4.

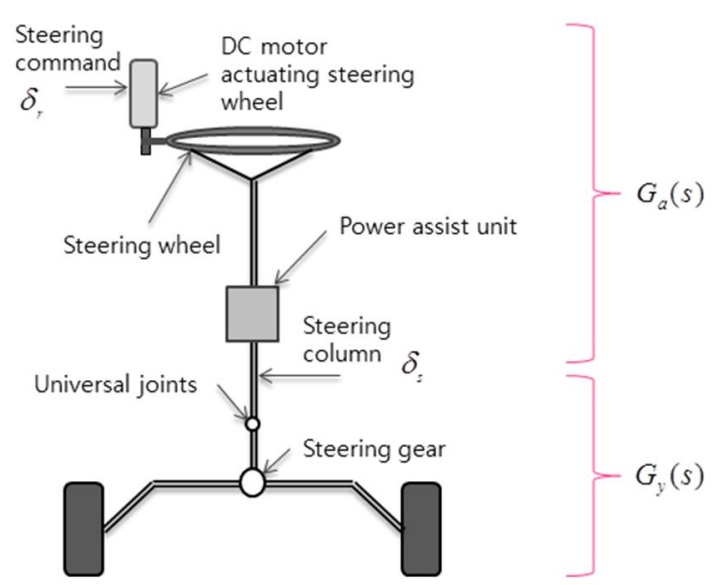

Fig. 3. Steering system representation

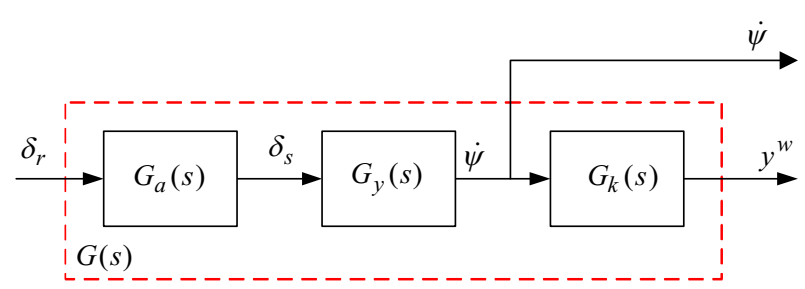

Fig. 4. Linear models for automatic steering system

The variables, $\delta_{r}$ and $\delta_{s}$, denote the reference steering angle and the angle of the steering column, respectively. $\dot{\psi}$ is the angular rate about the yaw axis, and $y^{w}$ is the lateral deviation of the vehicle body in the world coordinate.

From Fig. 4, the transfer function models are defined as follows;

$$
G_{a}:=\frac{\delta_{s}(s)}{\delta_{r}(s)}, \quad G_{y}:=\frac{\dot{\psi}(s)}{\delta_{s}(s)}, \quad G_{k}:=\frac{y^{w}(s)}{\dot{\psi}(s)} .
$$

The overall transfer function model from the steering command to the lateral deviation of vehicle can be represented as

$$
G(s):=\frac{y^{w}(s)}{\delta_{r}(s)}=G_{a}(s) G_{y}(s) G_{k}(s) .
$$

Since input and output variables, $\delta_{r}, \delta_{s,} \dot{\psi}$, and $y^{w}$ are available, it is possible to identify all the models in (1) and (2). The steering wheel actuator model $G_{a}(s)$ is a feedback system with a servo-motor controller, which will be explained in the subsection 3.3.1. The model $G_{a}(s)$ is useful when one deigns an actuator controller and evaluates its performance. The most important of vehicle motions to steering angle input is the yaw rate and lateral deviation of the body. These relationships are represented by the models, $G_{y}(s)$, and $G(s)$. Therefore, in this paper, we will show how to identify these three models, $G_{a}(s), G_{y}(s)$, and $G(s)$, using frequency response data. 


\subsection{Experimental inputs for frequency response data}

In order to obtain the frequency response data, we consider the following reference steering inputs, $\delta_{r}$, under a constant vehicle speed:

$$
\delta_{r}(t)=M_{k} \sin \left(\omega_{i} t\right), k=1, \cdots 4, i=1,2, \cdots, 8,
$$

where

- amplitudes $\left(M_{k}\right): \pm 30^{\circ}, 60^{\circ}, 90^{\circ}, 120^{\circ}$,

- frequencies $\left(\omega_{i}\right): 1,3,5,7,10,15,20,25[\mathrm{rad} / \mathrm{s}]$,

- vehicle speed: $30[\mathrm{~km} / \mathrm{h}]$,

- sampling frequency: $100 \mathrm{~Hz}$ for vehicle data and $20 \mathrm{~Hz}$ for GPS

The output variables, $\delta_{s}$ and $\dot{\psi}$, are transmitted from the built-in sensors of vehicle, while $y^{w}$ is estimated by using DGPS and IMU, which are equipped separately.

\subsection{Identifications and model validation}

\subsubsection{Actuator model: $\widehat{G}_{a}(s)$}

As explained in the previous subsection, the steering actuator has a feedback controller that makes the steering wheel angle track the steering command. This system can be represented by the feedback control system shown in Fig. 5.

When a steering command with amplitude of $M_{k}$ is applied, the corresponding frequency response of the closed-loop transfer function $G_{a}(s)=\delta_{s}(s) / \delta_{r}(s)$ is

$$
G_{a}^{k}\left(j \omega_{i}\right)=A_{i}^{k} \angle \emptyset_{i}^{k}, \quad i=1,2, \cdots, 8 \text { and } k=1,2,3,4
$$

where $A_{i}$ and $\emptyset_{i}$ denote the magnitude and phase responses of $G_{a}(s)$ at each frequency $\omega_{i}$.

Since the frequency response data must be acquired at steady state, the initial data of the measurements are not used. The magnitude $A_{i}$ was obtained by taking an average of several peaks of the sinusoidal responses. The phase $\phi_{i}$ was taken by the average of several phase differences between a sinusoidal input and its response at either peak or zero crossing time.

The experimental data are given in Tables A.1 - A.4 in Appendix A. For the given data, various orders of the models to be estimated are considered. By evaluating the

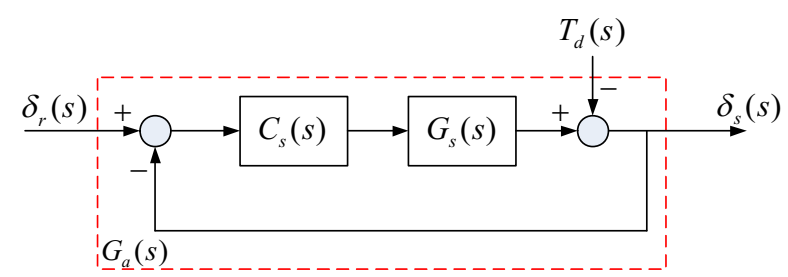

Fig. 5. Feedback system for steering actuator least squares criterion (as seen in (13)) for increasing estimated order, we have selected the following model structure as the best fit for those frequency responses.

$$
\hat{G}_{a}(s)=\frac{b_{0}}{s^{4}+a_{3} s^{3}+a_{2} s^{2}+a_{1} s+a_{0}}
$$

We now use the method of least squares $[9,10]$ to identify the parameters of the model in (5). From (4) and (5), we have:

$$
\begin{gathered}
A_{i} \cos \phi_{i}+j A_{i} \sin \phi_{i}=\frac{b_{0}}{\omega_{i}^{4}-j a_{3} \omega_{i}^{3}-a_{2} \omega_{i}^{2}+j a_{1} \omega_{i}+a_{0}}, \\
\text { for } i=1,2, \cdots, N,
\end{gathered}
$$

where $N$ is the number of observation.

Equating the real and imaginary parts of (6) separately and rearranging those yields:

$$
X_{i} \theta=Y_{i}, \text { for } i=1,2, \cdots, N \text {, }
$$

where

$$
\begin{aligned}
& \theta^{T}=\left[\begin{array}{lllll}
a_{0} & a_{1} & a_{2} & a_{3} & b_{0}
\end{array}\right], \\
& X_{i}^{T}=\left[\begin{array}{cc}
A_{i} \cos \phi_{i} & A_{i} \sin \phi_{i} \\
-\omega_{i} A_{i} \sin \phi_{i} & \omega_{i} A_{i} \cos \phi_{i} \\
-\omega_{i}^{2} A_{i} \cos \phi_{i} & -\omega_{i}^{2} A_{i} \sin \phi_{i} \\
\omega_{i}^{3} A_{i} \sin \phi_{i} & -\omega_{i}^{3} A_{i} \cos \phi_{i} \\
-1 & 0
\end{array}\right] \text {, } \\
& Y_{i}=\left[\begin{array}{ll}
-\omega_{i}^{4} A_{i} \cos \phi_{i} & -\omega_{i}^{4} A_{i} \sin \phi_{i}
\end{array}\right] \text {. }
\end{aligned}
$$

Let the resulting matrices be

$$
\begin{gathered}
X:=\left[\begin{array}{lllll}
X_{1} & X_{2} & X_{3} & \cdots & X_{N}
\end{array}\right]^{T}, \\
Y:=\left[\begin{array}{lllll}
Y_{1} & Y_{2} & Y_{3} & \cdots & Y_{N}
\end{array}\right]^{T} .
\end{gathered}
$$

The least squares criterion aims to minimize the following squared errors:

$$
J(\hat{\theta}):=\frac{1}{2}(Y-X \hat{\theta})^{T}(Y-X \hat{\theta}),
$$

where $\hat{\theta}$ indicates the estimated value of the parameter vector $\theta$. Then the least squares solution to (13) is given by

$$
\hat{\theta}=\left(X^{T} X\right)^{-1} X^{T} Y
$$

Substituting the data from Tables A.1 - A.4 into (14), we obtain four sets of identified values for the parameters of (5), as shown in Table 1 . That is, $\widehat{G_{s}^{k}}(s)$ for $k=1,2,3,4$ are the identified models corresponding to Table A.1, 2, 3, 
Table 1. Results of Identification for $\widehat{\boldsymbol{G}}_{\boldsymbol{s}}(\boldsymbol{s})$

\begin{tabular}{c|c|c|c|c|c}
\hline$\widehat{\theta}$ & $\widehat{G_{s}^{1}}(s)$ & $\widehat{G_{s}^{2}}(s)$ & $\widehat{G_{s}^{3}}(s)$ & $\widehat{G_{s}^{4}}(s)$ & Average \\
\hline$a_{0}$ & 76066 & 44096 & 32470 & 24519 & 44288 \\
\hline$a_{1}$ & 11510 & 6395.1 & 6004.3 & 4797.9 & 7176.8 \\
\hline$a_{2}$ & 895.39 & 805.92 & 788.1 & 738.28 & 806.92 \\
\hline$a_{3}$ & 30.22 & 21.09 & 21.296 & 18.018 & 22.66 \\
\hline$b_{0}$ & 66166 & 35051 & 26504 & 17742 & 36369 \\
\hline
\end{tabular}
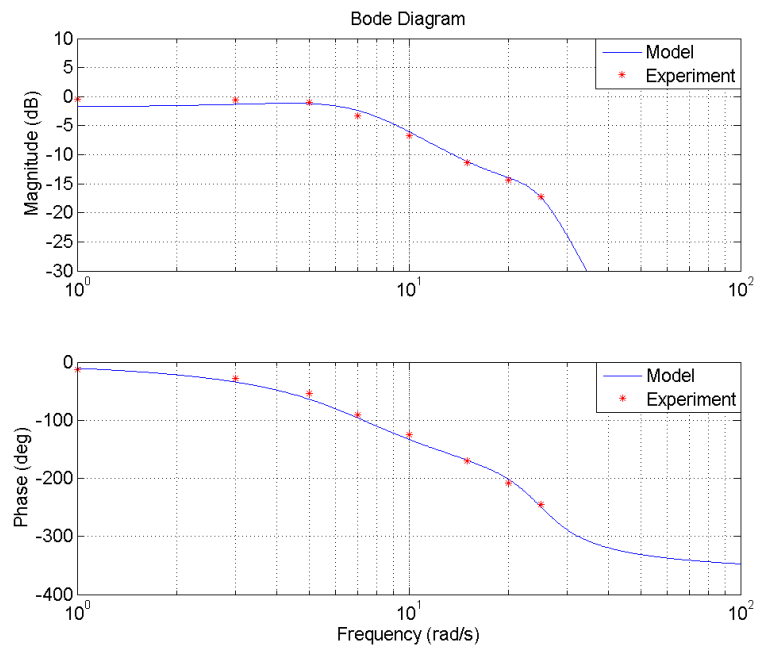

Fig. 6. Bode Plot of $\widehat{\boldsymbol{G}_{\boldsymbol{s}}^{\mathbf{3}}}(\boldsymbol{s})$ with Table A.1 data: model (blue line) and observations (red star)

and 4, respectively. Fig. 6 shows the Bode plot revealing the fitness of estimated model $\widehat{G_{s}^{3}}(s)$ with the measured data in Table A.3. The first break frequencies of the four models $\widehat{G_{s}^{k}}(s)$ for $k=1,2,3,4$ are about 7, 7, 5, and 5 $\mathrm{rad} / \mathrm{sec}$, respectively. We can also see from Table 1 that actuator models vary slightly depending on the amplitude of the operating angle of the steering wheel. Bode plots of the other models in Table 1 are very similar to the result of $\widehat{G_{S}^{3}}(s)$. Details are referred to in [11].

\subsubsection{Model between steering angle and yaw rate: $\widehat{G}_{y}(s)$}

When we apply the same steering inputs as the previous subsection 3.3.1, we obtain the frequency response data of the yaw rate model $G_{y}(s)$. The magnitude and phase data of $G_{y}^{k}\left(j \omega_{i}\right)=A_{i}^{k} \angle \phi_{i}^{k}, i=1,2, \cdots, 8, k=1,2,3,4$, are given in Tables B.1 - B.4 in Appendix B. As in the previous case, we have selected the following model structure for those frequency responses as the best fit:

$$
\widehat{G}_{y}(s)=\frac{b_{0}}{s^{3}+a_{2} s^{2}+a_{1} s+a_{0}}
$$

Similarly applying the least square method used for the identification of $\hat{G}_{y}(s)$ to model (15), we have 4 sets of parameter estimates, as shown in Table 2.

In Fig. 7, Bode plot of the average one of the four
Table 2. Results of Identification for $\widehat{G}_{y}(\boldsymbol{s})$

\begin{tabular}{c|c|c|c|c|c}
\hline$\hat{\theta}$ & $\widehat{G_{y}^{1}}(s)$ & $\widehat{G_{y}^{2}}(s)$ & $\widehat{G_{y}^{3}}(s)$ & $\widehat{G_{y}^{4}}(s)$ & Average \\
\hline$a_{0}$ & 10315 & 4470 & 4132 & 8873 & 6947 \\
\hline$a_{1}$ & 804 & 630 & 570 & 757 & 690 \\
\hline$a_{2}$ & 34.16 & 14.89 & 15.81 & 26.78 & 22.91 \\
\hline$b_{0}$ & 2173 & 1113 & 979.5 & 1672 & 1484 \\
\hline
\end{tabular}
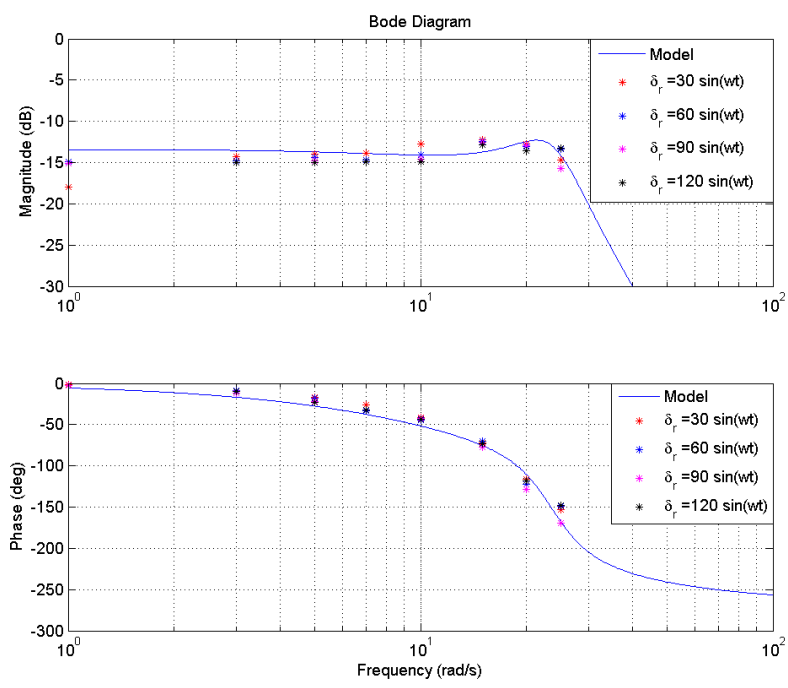

Fig. 7. Bode Plots of the average model $\widehat{\boldsymbol{G}}_{\boldsymbol{y}}(\boldsymbol{s})$ with observation data in Tables B.1-B.4

identified models $\widehat{G}_{y}(s)$ is compared with the observation data in Tables B.1 - B.4. The result shows that the identified yaw rate model is validated in a wide range of steering magnitudes and frequencies.

\subsubsection{Overall steering system model: $\hat{G}(s)$}

As shown in Fig.4, the overall steering system model is represented by a transfer function from the steering command to the lateral deviation of the vehicle. Frequency response data are collected when the steering command is $\delta_{r}(t)=120^{\circ} \sin \left(\omega_{i} t\right)$ and vehicle speed is $30 \mathrm{~km} / \mathrm{h}$. The data are given in Table C.1.

According to the well-known Bode's gain-phase theorem, the phase of any minimum phase system is approximately equal to $n_{s}$ times $90^{\circ}$, where $n_{s}$ is the slope of the magnitude curve in units of decade of amplitude per decade of frequency. If a system has a time delay $\left(T_{d}\right)$, the phase decreases by $-\omega T_{d}$. It is seen from Fig. 8 that the phase of $G(j \omega)$ to the magnitude slope around low frequencies is larger than the expected minimum phase system. The phase of $G(j \omega)$ shall be physically $-\pi \mathrm{rad}$ at $\omega=0$. We regarded the phase difference at $\omega=3$ as the effect of delay. Thus, the time delay was estimated as

$$
T_{d}=(-\pi+3.48) / 3=0.1128 \mathrm{sec} .
$$



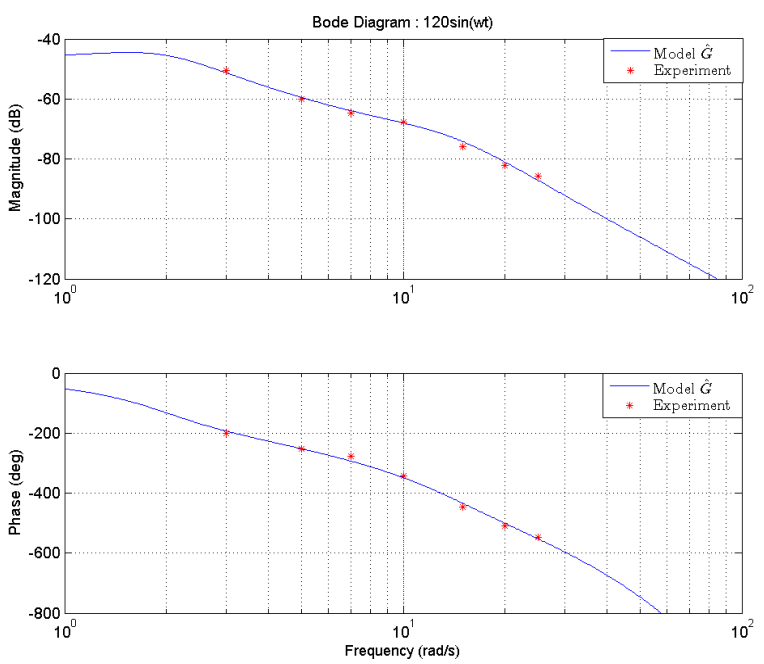

Fig. 8. Bode plot of the identified overall model with measurements in Table C. 1

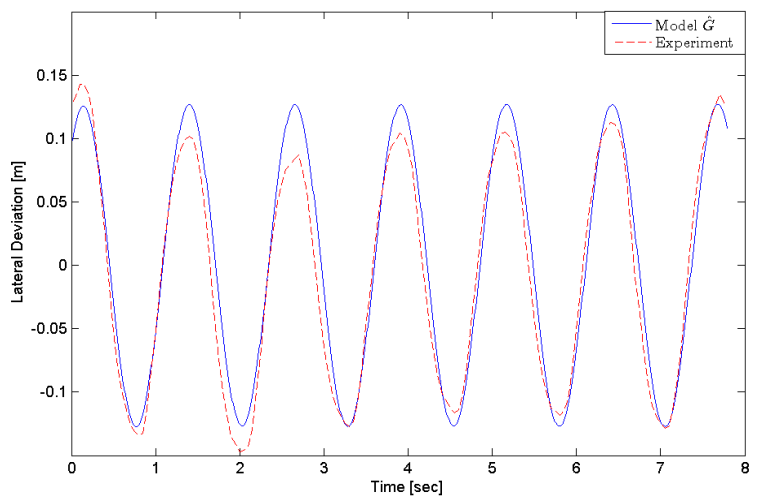

(a) $\delta_{r}(t)=120^{\circ} \sin \left(\omega_{3} t\right), \omega_{3}=5 \mathrm{rad} / \mathrm{s}$

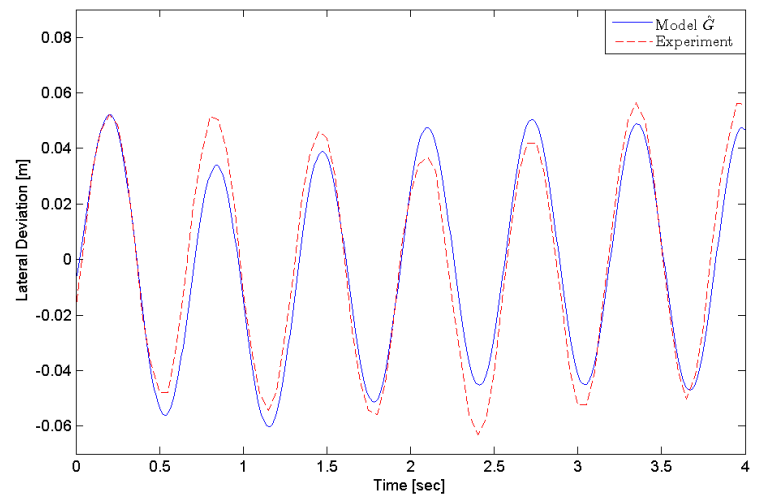

(b) $\delta_{r}(t)=120^{\circ} \sin \left(\omega_{5} t\right), \omega_{5}=10 \mathrm{rad} / \mathrm{s}$

Fig. 9. Output responses of the identified model $\hat{G}(s)$ and the steering system of vehicle to two sinusoidal test inputs

Applying the identification method in the previous section again results in the following model:

$$
\hat{G}(s)=\frac{-0.5953 s+3.554}{s^{4}+16.54 s^{3}+231.2 s^{2}+413 s+768} e^{-0.1128 s}
$$

As shown in Fig. 8, the Bode plot of the identified model $\hat{G}(s)$ coincides with the measurement data in Table C. 1 . In many cases, the validity test of modeling based on frequency response is sufficient to compare the measured data and the model in the Bode diagram. However, when one wants to design a steering controller for an autonomous vehicle, time response behavior as well as frequency response of the model may be required. Thus, the output responses of the overall steering system to two sinusoidal test inputs are also compared in Fig. 9 by depicting both the output of the identified model and the measured data from vehicle in time domain. As a result, we can see from Fig. 8 and 9 that model (16) is well fitted for the overall steering system.

\section{Conclusion}

Autonomous vehicles require a lateral guidance controller with high performance. To design such a controller, it is necessary to have a proper steering system model. This paper suggests an experimental modeling approach based on the frequency response under a constant speed condition. We show that the overall model structure for a steering system can be represented by three transfer function models. Experimental tests were carried out with a real SUV vehicle, and the parameters of the model set were identified using the least squares estimation (LSE) method. It was shown through Bode plots and time responses that the identified models match the observation data well. This modeling approach can be highly useful for the practical design of steering controllers in particular for cases where classical controllers such as PID, first-order controllers are used.

\section{Acknowledgements}

This research was supported by Basic Science Research Program through the National Research Foundation of Korea (NRF) funded by the Ministry of Education (No. NRF-2015R1D1A01060997) and was conducted during the research year of Chungbuk National University in 2015 .

\section{References}

[1] J. Ackermann, J. Guldner, W. Sienel, R. Steinhauser, and V. I. Utkin, "Linear and Nonlinear Controller Design for Robust Automatic Steering," IEEE Trans. on Control System Technology, vol. 3, no. 1, pp. 132- 
142,1995

[2] P. Falcone, F. Borrelli, J. Asgari, H.E. Tseng, and D. Hrovat "Predictive Active Steering Control for Autonomous Vehicle Systems," IEEE Trans. on Control Systems Technology, vol. 15, no. 3, pp. 566580, 2007.

[3] Y. C. Kim, K. Yun, and K. Min, "Automatic guidance control of an articulated-all wheel steered vehicle," Vehicle System Dynamics, vol. 52, no. 4, pp. 456-474, 2014.

[4] D. Minoiu Enache, M. Netto, S. Mammar, and B. Lusetti, "Driver steering assistance for lane departure avoidance," Control Engineering Practice, vol. 17, pp. 642-651, 2009.

[5] M. Alirezaei, M. Corno, A. Ghaffari, and R. Kazemi, "A Robust Steering Assistance System for Road Departure Avoidance," IEEE Trans. on Vehicular Technology, vol. 61, no. 5, pp. 1953-1960, 2012

[6] R. Rajamani, Vehicle Dynamics and Control, Springer Verlag, 2005.

[7] K. Min and Y.C. Kim, "Modeling and Validation of 16 DOF Full Vehicle Model for Guidance Control," International Journal of Vehicle Systems Modeling and Testing, vol. 10, no. 4, pp. 392-416, 2015.

[8] Y. C. Kim, K. H. Yun, K. Min, Y. S. Byun, and J. K. Mok, "A Lateral Dynamic Model of an All Wheel Steered Bimodal Vehicle," in Proc. of Int'l Conf. on Control, Automation, and Systems, pp. 1734-1737, Seoul Korea, Oct. 2007

[9] R. Johansson, System Modeling and Identification, Prentice-Hall International Inc., New Jersey, 1993.

[10] A. Datta, et al. 5, "Design and Implementation of a Controller for the SBW system," Technical Report, University of Southern California, 1989.

[11] J. Kim, Controller Design for the Steering System of Autonomous Vehicle, MS thesis, Chungbuk National University, Korea, Feb. 2014.

\section{Appendix}

A. Frequency responses data for $G_{a}(s)$ at a constant speed of $30 \mathrm{~km} / \mathrm{h}$.

Table A.1. Steering command: $\delta_{r}(t)=30^{\circ} \sin \left(\omega_{i} t\right)$,

\begin{tabular}{c|c|c|c|c|c|c|c|c}
\hline$\omega[\mathrm{rad} / s]$ & 1 & 3 & 5 & 7 & 10 & 15 & 20 & 25 \\
\hline$A_{i}$ & 26.8 & 26.3 & 25.3 & 24.2 & 23.3 & 20.4 & 15.2 & 10.4 \\
\hline$-\emptyset_{i}[\mathrm{rad}]$ & 0.29 & 0.56 & 0.85 & 1.08 & 1.48 & 2.39 & 3.36 & 4.16 \\
\hline
\end{tabular}

Table A.2. Steering command: $\delta_{r}(t)=60^{\circ} \sin \left(\omega_{i} t\right)$,

\begin{tabular}{c|c|c|c|c|c|c|c|c}
\hline$\omega[\mathrm{rad} / s]$ & 1 & 3 & 5 & 7 & 10 & 15 & 20 & 25 \\
\hline$A_{i}$ & 56.4 & 55.4 & 53.6 & 52.3 & 38.4 & 22.3 & 15.6 & 12.3 \\
\hline$-\emptyset_{i}[\mathrm{rad}]$ & 0.24 & 0.51 & 0.76 & 1.21 & 1.96 & 2.95 & 3.63 & 4.21 \\
\hline
\end{tabular}

Table A.3. Steering command: $\delta_{r}(t)=90^{\circ} \sin \left(\omega_{i} t\right)$,

\begin{tabular}{c|c|c|c|c|c|c|c|c}
\hline$\omega[\mathrm{rad} / s]$ & 1 & 3 & 5 & 7 & 10 & 15 & 20 & 25 \\
\hline$A_{i}$ & 86.2 & 84.2 & 80.2 & 61.1 & 41.4 & 24.3 & 17.1 & 12.4 \\
\hline$-\emptyset_{i}[\mathrm{rad}]$ & 0.22 & 0.5 & 0.95 & 1.58 & 2.19 & 2.98 & 3.63 & 4.27 \\
\hline
\end{tabular}

Table A.4. Steering command: $\delta_{r}(t)=120^{\circ} \sin \left(\omega_{i} t\right)$,

\begin{tabular}{c|c|c|c|c|c|c|c|c}
\hline$\omega[\mathrm{rad} / s]$ & 1 & 3 & 5 & 7 & 10 & 15 & 20 & 25 \\
\hline$A_{i}$ & - & 113. & 88.2 & 63.0 & 42 & 24.6 & 17.7 & 13.2 \\
\hline$-\emptyset_{i}[\mathrm{rad}]$ & - & 0.48 & 1.28 & 1.8 & 2.32 & 3.1 & 3.8 & 4.2 \\
\hline
\end{tabular}

B. Frequency responses data for $G_{y}(s)$ at a constant speed of $30 \mathrm{~km} / \mathrm{h}$.

Table B.1. Steering command: $\delta_{r}(t)=30^{\circ} \sin \left(\omega_{i} t\right)$,

\begin{tabular}{c|c|c|c|c|c|c|c|c}
\hline$\omega[\mathrm{rad} / s]$ & 1 & 3 & 5 & 7 & 10 & 15 & 20 & 25 \\
\hline$A_{i}$ & 0.13 & 0.19 & 0.2 & 0.2 & 0.23 & 0.25 & 0.23 & 0.18 \\
\hline$-\emptyset_{i}[\mathrm{rad}]$ & 0.03 & 0.16 & 0.29 & 0.45 & 0.72 & 1.26 & 2.02 & 2.68 \\
\hline
\end{tabular}

Table B.2. Steering command: $\delta_{r}(t)=60^{\circ} \sin \left(\omega_{i} t\right)$,

\begin{tabular}{c|c|c|c|c|c|c|c|c}
\hline$\omega\left[{ }^{\mathrm{rad}} / s\right]$ & 1 & 3 & 5 & 7 & 10 & 15 & 20 & 25 \\
\hline$A_{i}$ & 0.18 & 0.18 & 0.19 & 0.18 & 0.2 & 0.24 & 0.22 & 0.21 \\
\hline$-\emptyset_{i}[\mathrm{rad}]$ & 0.05 & 0.14 & 0.31 & 0.59 & 0.78 & 1.23 & 2.14 & 2.6 \\
\hline
\end{tabular}

Table B.3. Steering command: $\delta_{r}(t)=90^{\circ} \sin \left(\omega_{i} t\right)$,

\begin{tabular}{c|c|c|c|c|c|c|c|c}
\hline$\omega[\mathrm{rad} / s]$ & 1 & 3 & 5 & 7 & 10 & 15 & 20 & 25 \\
\hline$A_{i}$ & 0.17 & 0.18 & 0.18 & 0.18 & 0.19 & 0.24 & 0.22 & 0.16 \\
\hline$-\emptyset_{i}[\mathrm{rad}]$ & 0.05 & 0.2 & 0.39 & 0.57 & 0.74 & 1.35 & 2.24 & 2.95 \\
\hline
\end{tabular}

Table B.4. Steering command: $\delta_{r}(t)=120^{\circ} \sin \left(\omega_{i} t\right)$,

\begin{tabular}{c|c|c|c|c|c|c|c|c}
\hline$\omega[\mathrm{rad} / s]$ & 1 & 3 & 5 & 7 & 10 & 15 & 20 & 25 \\
\hline$A_{i}$ & - & 0.18 & 0.18 & 0.18 & 0.18 & 0.23 & 0.21 & 0.21 \\
\hline$-\emptyset_{i}[\mathrm{rad}]$ & - & 0.17 & 0.41 & 0.56 & 0.76 & 1.28 & 2.06 & 2.58 \\
\hline
\end{tabular}

C. Frequency responses data for $G(s)$ at a constant speed of $30 \mathrm{~km} / \mathrm{h}$.

Table C.1. Steering command: $\delta_{r}(t)=120^{\circ} \sin \left(\omega_{i} t\right)$,

\begin{tabular}{c|c|c|c|c|c|c|c|c}
\hline$\omega[\mathrm{rad} / s]$ & 1 & 3 & 5 & 7 & 10 & 15 & 20 & 25 \\
\hline$A_{i}\left(\mathrm{x} 10^{-3}\right)$ & - & 3.015 & 0.9958 & 0.5692 & 0.4175 & 0.1608 & 0.0775 & 0.0508 \\
\hline$-\emptyset_{i}[\mathrm{rad}]$ & - & 3.48 & 4.4 & 4.83 & 6 & 7.783 & 8.883 & 9.533 \\
\hline
\end{tabular}




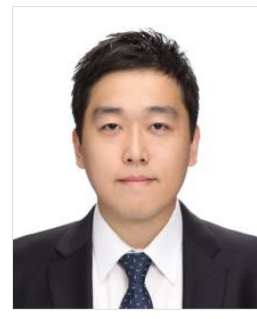

Ju-Young Kim He received B.S. and M.S. degrees in Electronics Eng. from Chungbuk National University, in 20 12 and 2014, respectively. Mr. Kim and his colleagues received a second prize at the $11^{\text {th }}$ Hyundai Motor Group Autonomous Vehicle Competition in 2012 and the winner of the 3rd TI Korea MCU Design Contest. He is currently working for LG Uplus Corp., Korea, as an assistant manager since 2004.

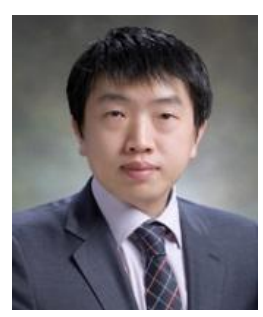

Kyungdeuk Min He received his B.S., M.S. and Ph.D. degrees in Electronics Eng. from the Chungbuk National University (CBNU), Korea, in 2007, 2009, and 2014, respectively. Dr. Min was the leader of the second prize team at the $11^{\text {th }}$ Hyundai Motor Group Autonomous Vehicle Competition in 2012 and has been recognized by multiple awards at many other competitions in Korea. He is currently a senior research engineer at the ADAS Engineering Design Team, Automotive Research \& Development Division, Hyundai Motor Group. His research interests include dynamic system modeling and ADAS designs for autonomous vehicle.

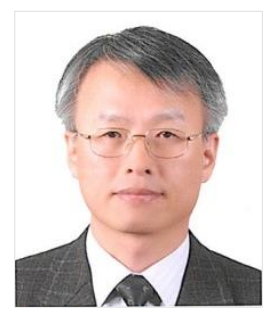

Young Chol Kim He received the B.S. from the Korea University, Seoul, Korea in 1981, M.S. and Ph.D. degrees in Electrical Eng. from the Seoul National University, Seoul Korea, in 1983 and 1987, respectively. He has been with the Department of Electronics Eng., Chungbuk National University, Korea since 1988 and is currently a Professor. He was a visiting scholar at Texas A \& M University in 1991 and Tennessee State University in 2001. He served the president of the Information and Control Society of the Korean Institute of Electrical Engineers from 2009 to 2010. Dr. Kim received Myungsam Ko award from ICROS in 2004, Heungseok Yang award from KIEE in 2012, and multiple awards for outstanding papers. His research interests are in the areas of parametric robust control, dynamic system modeling, low-order controller design for practical industry plants, control system designs for autonomous vehicle, EV, and $\mathrm{HEV}$. 